\title{
COMPOSITE QUASIANALYTIC FUNCTIONS
}

\author{
ANDRÉ BELOTTO DA SILVA, EDWARD BIERSTONE, AND MICHAEL CHOW
}

\begin{abstract}
We prove two main results on Denjoy-Carleman classes: (1) a composite function theorem which asserts that a function $f(x)$ in a quasianalytic Denjoy-Carleman class $\mathcal{Q}_{M}$, which is formally composite with a generically submersive mapping $y=\varphi(x)$ of class $\mathcal{Q}_{M}$, at a single given point in the source (or in the target) of $\varphi$ can be written locally as $f=g \circ \varphi$, where $g(y)$ belongs to a shifted Denjoy-Carleman class $\mathcal{Q}_{M(p)} ;(2)$ a statement on a similar loss of regularity for functions definable in the $o$-minimal structure given by expansion of the real field by restricted functions of quasianalytic class $\mathcal{Q}_{M}$. Both results depend on an estimate for the regularity of a $\mathcal{C}^{\infty}$ solution $g$ of the equation $f=g \circ \varphi$, with $f$ and $\varphi$ as above. The composite function result depends also on a quasianalytic continuation theorem, which shows that the formal assumption at a given point in (1) propagates to a formal composition condition at every point in a neighbourhood.
\end{abstract}

\section{Contents}

1. Introduction

2. Quasianalytic classes

3. Quasianalytic continuation

4. Proofs of the main theorems 9

5. Regularity estimates 10

References

\section{INTRODUCTION}

This article contains two main results on Denjoy-Carleman classes:

(1) a composite function theorem which asserts that a function $f(x)$ in a quasianalytic Denjoy-Carleman class $\mathcal{Q}_{M}$, which is formally composite with a generically submersive mapping $y=\varphi(x)$ of class $\mathcal{Q}_{M}$, at a given point $x=a$ (or at a given point $y=b$ ) can be written locally as $f=g \circ \varphi$, where $g(y)$ belongs to a shifted Denjoy-Carleman class $\mathcal{Q}_{M^{(p)}}$ (see Theorems 1.1 and 1.2 for precise versions of this assertion that are local at $a$ or $b$, respectively);

Date: September 21, 2017.

2010 Mathematics Subject Classification. Primary 03C64, 26E10, 32S45; Secondary 30D60, 32B20.

Key words and phrases. quasianalytic, Denjoy-Carleman class, o-minimal structure, resolution of singularities, composite function theorem, quasianalytic continuation.

Research supported by CIMI LabEx (Belotto), NSERC OGP0009070 (Bierstone), NSERC USRA and OGP0009070 (Chow). 
(2) a statement on a similar loss of regularity for functions definable in the ominimal structure given by expansion of the real field by restricted functions of quasianalytic class $\mathcal{Q}_{M}$ (Theorem 1.6).

Both results depend on an estimate for the regularity of a $\mathcal{C}^{\infty}$ solution $g$ of the equation $f=g \circ \varphi$, with $f$ and $\varphi$ as above (Theorem 1.4). The composite function result depends also on a quasianalytic continuation theorem (Theorem 1.3 below), which shows that the formal assumption at a given point in (1) propagates to a formal composition condition at every point in a neighbourhood.

Quasianalytic Denjoy-Carleman classes go back to E. Borel [6] and were characterized (following questions of Hadamard in studies of linear partial differential equations [11]) by the Denjoy-Carleman theorem 9], 77. Quasianalytic classes arise in model theory as the classes of $\mathcal{C}^{\infty}$ functions that are definable in a given polynomially-bounded o-minimal structure [17, [21]. Relevant background on Denjoy-Carleman and quasianalytic classes is presented in Section 2 below.

Given a $\mathcal{C}^{\infty}$ function $f(x)$ or a $\mathcal{C}^{\infty}$ mapping $y=\varphi(x)$ defined on an open subset of $\mathbb{R}^{n}$, we write $\hat{f}_{a}$ or $\hat{\varphi}_{a}$ for the formal Taylor expansions at a point $x=a$.

Theorem 1.1. Let $\mathcal{Q}_{M}$ denote a quasianalytic Denjoy-Carleman class, and let $\varphi: V \rightarrow W$ be a mapping of class $\mathcal{Q}_{M}$ between open sets $V \subset \mathbb{R}^{m}$ and $W \subset \mathbb{R}^{n}$, which is generically a submersion (i.e., generically of rank $n$ ). Let $f \in \mathcal{Q}_{M}(V)$. If $a \in V$ and $\hat{f}_{a}=G \circ \hat{\varphi}_{a}$, where $G$ is a formal power series centred at $\varphi(a)$, then there is a relatively compact neighbourhood $U$ of a in $V$ and a function $g \in \mathcal{Q}_{M^{(p)}}(\varphi(\bar{U}))$, for some $p \in \mathbb{N}$, such that $f=g \circ \varphi$ on $U$.

Theorem 1.2. Let $\mathcal{Q}_{M}$ denote a quasianalytic Denjoy-Carleman class, and let $\varphi: V \rightarrow W$ be a $\mathcal{Q}_{M}$-mapping of $\mathcal{Q}_{M}$-manifolds which is proper and generically submersive. Let $f \in \mathcal{Q}_{M}(V)$ and let $b \in \varphi(V)$. Suppose that $\hat{f}_{a}=G \circ \hat{\varphi}_{a}$, for all $a \in \varphi^{-1}(b)$, where $G$ is a formal power series centred at $b$. Then, after perhaps shrinking $W$ to an appropriate neighbourhood of b, there exists $g \in \mathcal{Q}_{M^{(p)}}(\varphi(V))$, for some $p$, such that $f=g \circ \varphi$.

The formal expansions in Theorem 1.2 can be defined using local coordinate systems, and the condition $\hat{f}_{a}=G \circ \hat{\varphi}_{a}$ is independent of the choice of local coordinates at $a$ and $b$.

We recall that in Glaeser's $\mathcal{C}^{\infty}$ composition theorem [10, where $\varphi$ is real-analytic, $f$ is $\mathcal{C}^{\infty}$ and we seek a $\mathcal{C}^{\infty}$ solution $g$, it is necessary to assume the existence of a formal solution at every point of the target. It is striking that, as a result of quasianalytic continuation, it is sufficient to assume there is a formal solution at a single point in Theorems 1.1 and 1.2. In particular, Theorem 1.1 reduces to the classical statement in the real-analytic case (cf. [2, Lemma 7.8], [15]), even though it seems unlikely that the ring of formal power series at a point is flat over the local ring of germs of functions of class $\mathcal{Q}_{M}$, in general.

The main ingredients in our proofs of Theorems 1.1 1.2 are Theorems 1.3 and 1.4, and a quasianalytic version [18] of Glaeser's theorem [10]. It follows from Theorem 1.3 that Theorems 1.1 and 1.2 are equivalent (see Remark 4.1) and also that, in Theorem 1.2, we can weaken the assumption to $\hat{f}_{a}=G \circ \hat{\varphi}_{a}$, for a single point $a$ in each connected component of $\varphi^{-1}(b)$.

1.1. Quasianalytic continuation. The following theorem is a further development of quasianalytic continuation techniques introduced in [1, Sect. 4]. 
Theorem 1.3. Let $\mathcal{Q}$ denote a quasianalytic class (see Definition[2.1). Let $\varphi: V \rightarrow$ $W$ denote a $\mathcal{Q}$-mapping, where $V$ is a $\mathcal{Q}$-manifold and $W$ is an open neighbourhood of $0 \in \mathbb{R}^{n}$. Let $f \in \mathcal{Q}(V)$ and let $H$ denote a formal power series at $0 \in \mathbb{R}^{n}$. Then:

(1) $\left\{a \in \varphi^{-1}(0): \hat{f}_{a}=H \circ \hat{\varphi}_{a}\right\}$ is open and closed in $\varphi^{-1}(0)$.

(2) Suppose that $\varphi$ is proper and generically of rank $n$. Assume $\hat{f}_{a}=H \circ \hat{\sigma}_{a}$, for all $a \in \varphi^{-1}(0)$. Then, after perhaps shrinking $W, f$ is formally composite with $\varphi$; i.e., for all $b \in W$, there exists a power series $H_{b}$ centred at $b$ such that $\hat{f}_{a}=H_{b} \circ \hat{\varphi}_{a}^{*}$, for all $a \in \varphi^{-1}(b)$.

The composite function theorem 1.2 was proved by Chaumat and Chollet $[8]$ in the special case that $\varphi$ is a real-analytic mapping, under the stronger assumption that $f$ is formally composite with $\varphi$ as in the conclusion of Theorem 1.3(2) (i.e., throughout the image of $\varphi$, as in Glaeser's theorem). Theorem 1.4 below is a generalization to a mapping in a given Denjoy-Carleman class of an estimate for a real-analytic mapping in [8, Sect. III, Prop. 8].

1.2. Regularity estimates. Theorem[1.4 and Corollary 1.5]following will be proved in Section 4. Our proof of Theorem 1.4 follows the reasoning used in [1, Lemmas $3.1,3.4$, which give more precise estimates in the special cases that $\sigma$ is a power substitution or a blowing-up. See Section 2 for the notation used in the theorem.

Theorem 1.4. Let $\mathcal{Q}_{M}$ denote a Denjoy-Carleman class (see Definition [2.3). Let $\sigma: V \rightarrow W$ denote a mapping of class $\mathcal{Q}_{M}$ between open subsets of $\mathbb{R}^{n}$, such that the Jacobian determinant $\operatorname{det}(\partial \sigma / \partial x)$ is a monomial $x^{\gamma}=x_{1}^{\gamma_{1}} \cdots x_{n}^{\gamma_{n}}$ times a nowhere vanishing factor. Let $g \in \mathcal{C}^{\infty}(W)$ and assume that $f:=g \circ \sigma \in \mathcal{Q}_{M}(V)$. Then, for every compact $K \subset V, g$ is of class $\mathcal{Q}_{M^{(p)}}$ on $\sigma(K)$, where

$$
p:=2|\gamma|+1
$$

i.e., there exist constants $A, B>0$ such that

$$
\left|\frac{\partial^{|\beta|} g}{\partial y^{\beta}}\right| \leq A B^{|\beta|} \beta ! M_{p|\beta|} \quad \text { on } \sigma(K), \text { for every } \beta \in \mathbb{N}^{n} .
$$

Moreover, if $\mathcal{Q}_{M}$ is closed under differentiation (in particular, if $\mathcal{Q}_{M}$ is a quasianalytic Denjoy-Carleman class; see Section 圆, then we can take

$$
p=|\gamma|+1 \text {. }
$$

Corollary 1.5. Let $\mathcal{Q}_{M}$ denote a quasianalytic Denjoy-Carleman class, and let $\varphi: V \rightarrow W$ be a proper (or semiproper), generically submersive mapping of class $\mathcal{Q}_{M}$, where $V$ and $W$ are $\mathcal{Q}_{M}$-manifolds. Let $g \in \mathcal{C}^{\infty}(W)$. If $f:=g \circ \varphi \in \mathcal{Q}_{M}(V)$, then, for every relatively compact open $U \subset W$, there exists $p \in \mathbb{N}$ (depending only on $\varphi$ and $U)$ such that $g \in \mathcal{Q}_{M^{(p)}}(\varphi(V) \cap U)$.

1.3. Model theory. Let $\mathcal{Q}_{M}$ denote a quasianalytic Denjoy-Carleman class, and let $\mathbb{R}_{\mathcal{Q}_{M}}$ denote the expansion of the real field by restricted functions of class $\mathcal{Q}_{M}$ (i.e., restrictions to closed cubes of $\mathcal{Q}_{M}$-functions, extended by 0 outside the cube). Then $\mathbb{R}_{\mathcal{Q}_{M}}$ is an o-minimal structure, and $\mathbb{R}_{\mathcal{Q}_{M}}$ is both polynomially bounded and model-complete [21].

By [17, the class of $\mathcal{C}^{\infty}$ functions that are definable in any given polynomially bounded $o$-minimal structure satisfy the quasianalyticity property (Definition 2.1(3)). The following will be proved in Section 3, using the regularity estimate above. 
Theorem 1.6. Let $\mathcal{Q}_{M}$ denote a quasianalytic Denjoy-Carleman class. If $f \in$ $\mathcal{C}^{\infty}(W)$, where $W$ is open in $\mathbb{R}^{n}$, and $f$ is definable in $\mathbb{R}_{\mathcal{Q}_{M}}$, then there exists $p \in \mathbb{N}$ such that $f \in \mathcal{Q}_{M^{(p)}}(W)$.

The graph of a function definable in $\mathbb{R}_{\mathcal{Q}_{M}}$ is sub-quasianalytic in the obvious sense generalizing subanalytic. If $f \in \mathcal{C}^{\infty}(W)$ has sub-quasianalytic graph, then $f \in \mathcal{Q}_{M^{(p)}}(U)$, for some $p=p_{U}$, for every relatively compact open $U \subset W$; in general, however, $\left\{p_{U}\right\}$ need not be bounded.

\section{Quasianalytic Classes}

We consider a class of functions $\mathcal{Q}$ given by the association, to every open subset $U \subset \mathbb{R}^{n}$, of a subalgebra $\mathcal{Q}(U)$ of $\mathcal{C}^{\infty}(U)$ containing the restrictions to $U$ of polynomial functions on $\mathbb{R}^{n}$, and closed under composition with a $\mathcal{Q}$-mapping (i.e., a mapping whose components belong to $\mathcal{Q}$ ). We assume that $\mathcal{Q}$ determines a sheaf of local $\mathbb{R}$-algebras of $\mathcal{C}^{\infty}$ functions on $\mathbb{R}^{n}$, for each $n$, which we also denote $\mathcal{Q}$.

Definition 2.1 (quasianalytic classes). We say that $\mathcal{Q}$ is quasianalytic if it satisfies the following three axioms:

(1) Closure under division by a coordinate. If $f \in \mathcal{Q}(U)$ and

$$
f\left(x_{1}, \ldots, x_{i-1}, a, x_{i+1}, \ldots, x_{n}\right)=0,
$$

where $a \in \mathbb{R}$, then $f(x)=\left(x_{i}-a\right) h(x)$, where $h \in \mathcal{Q}(U)$.

(2) Closure under inverse. Let $\varphi: U \rightarrow V$ denote a $\mathcal{Q}$-mapping between open subsets $U, V$ of $\mathbb{R}^{n}$. Let $a \in U$ and suppose that the Jacobian matrix $(\partial \varphi / \partial x)(a)$ is invertible. Then there are neighbourhoods $U^{\prime}$ of $a$ and $V^{\prime}$ of $b:=\varphi(a)$, and a $\mathcal{Q}$-mapping $\psi: V^{\prime} \rightarrow U^{\prime}$ such that $\psi(b)=a$ and $\psi \circ \varphi$ is the identity mapping of $U^{\prime}$.

(3) Quasianalyticity. If $f \in \mathcal{Q}(U)$ has Taylor expansion zero at $a \in U$, then $f$ is identically zero near $a$.

Remarks 2.2. (1) Axiom 2.1(1) implies that, if $f \in \mathcal{Q}(U)$, then all partial derivatives of $f$ belong to $\mathcal{Q}(U)$.

(2) Axiom 2.1(2) is equivalent to the property that the implicit function theorem holds for functions of class $\mathcal{Q}$. It implies that the reciprocal of a nonvanishing function of class $\mathcal{Q}$ is also of class $\mathcal{Q}$.

(3) Our two main examples of quasianalytic classes are quasianalytic DenjoyCarleman classes (see \$2.1), and the class of $\mathcal{C}^{\infty}$ functions definable in a given polynomially bounded $o$-minimal structure (4.2). In the latter case, we can define a quasianalytic class $\mathcal{Q}$ in the axiomatic framework above by taking $\mathcal{Q}(U)$ as the subring of $\mathcal{C}^{\infty}(U)$ of functions $f$ such that $f$ is definable in some neighbourhood of any point of $U$ (or, equivalently, such that $\left.f\right|_{V}$ is definable, for every relatively compact definable open $V \subset U$ ); the division and inverse properties are immediate from definability and the corresponding $\mathcal{C}^{\infty}$ assertions.

The elements of a quasianalytic class $\mathcal{Q}$ will be called quasianalytic functions. A category of manifolds and mappings of class $\mathcal{Q}$ can be defined in a standard way. The category of $\mathcal{Q}$-manifolds is closed under blowing up with centre a $\mathcal{Q}$ submanifold [4]. 
Resolution of singularities holds in a quasianalytic class [3], 4]. Resolution of singularities of an ideal does not require that the ideal be finitely generated; see [5, Thm. 3.1].

2.1. Quasianalytic Denjoy-Carleman classes. We use standard multiindex notation: Let $\mathbb{N}$ denote the nonnegative integers. If $\alpha=\left(\alpha_{1}, \ldots, \alpha_{n}\right) \in \mathbb{N}^{n}$, we write $|\alpha|:=\alpha_{1}+\cdots+\alpha_{n}, \alpha !:=\alpha_{1} ! \cdots \alpha_{n} !, x^{\alpha}:=x_{1}^{\alpha_{1}} \cdots x_{n}^{\alpha_{n}}$, and $\partial^{|\alpha|} / \partial x^{\alpha}:=$ $\partial^{\alpha_{1}+\cdots+\alpha_{n}} / \partial x_{1}^{\alpha_{1}} \cdots \partial x_{n}^{\alpha_{n}}$. We write $(i)$ for the multiindex with 1 in the $i$ th place and 0 elsewhere.

Definition 2.3 (Denjoy-Carleman classes). Let $M=\left(M_{k}\right)_{k \in \mathbb{N}}$ denote a sequence of positive real numbers which is logarithmically convex; i.e., the sequence $\left(M_{k+1} / M_{k}\right)$ is nondecreasing. A Denjoy-Carleman class $\mathcal{Q}=\mathcal{Q}_{M}$ is a class of $\mathcal{C}^{\infty}$ functions determined by the following condition: A function $f \in \mathcal{C}^{\infty}(U)$ (where $U$ is open in $\mathbb{R}^{n}$ ) is of class $\mathcal{Q}_{M}$ if, for every compact subset $K$ of $U$, there exist constants $A, B>0$ such that

$$
\left|\frac{\partial^{|\alpha|} f}{\partial x^{\alpha}}\right| \leq A B^{|\alpha|} \alpha ! M_{|\alpha|}
$$

on $K$, for every $\alpha \in \mathbb{N}^{n}$.

Remark 2.4. The logarithmic convexity assumption implies that $M_{j} M_{k} \leq M_{0} M_{j+k}$, for all $j, k$, and that the sequence $\left(\left(M_{k} / M_{0}\right)^{1 / k}\right)$ is nondecreasing. The first of these conditions guarantees that $\mathcal{Q}_{M}(U)$ is a ring, and the second that $\mathcal{Q}_{M}(U)$ contains the ring $\mathcal{O}(U)$ of real-analytic functions on $U$, for every open $U \subset \mathbb{R}^{n}$. (If $M_{k}=1$, for all $k$, then $\mathcal{Q}_{M}=\mathcal{O}$.)

If $X$ is a closed subset of $U$, then $\mathcal{Q}_{M}(X)$ will denote the ring of restrictions to $X$ of $\mathcal{C}^{\infty}$ functions which satisfy estimates of the form (2.1), for every compact $K \subset X$.

A Denjoy-Carleman class $\mathcal{Q}_{M}$ is a quasianalytic class in the sense of Definition 2.1 if and only if the sequence $M=\left(M_{k}\right)_{k \in \mathbb{N}}$ satisfies the following two assumptions in addition to those of Definition 2.3

$$
\begin{aligned}
& \text { (a) } \sup \left(\frac{M_{k+1}}{M_{k}}\right)^{1 / k}<\infty . \\
& \text { (b) } \sum_{k=0}^{\infty} \frac{M_{k}}{(k+1) M_{k+1}}=\infty .
\end{aligned}
$$

It is easy to see that the assumption (a) implies that $\mathcal{Q}_{M}$ is closed under differentiation. The converse of this statement is due to S. Mandelbrojt [16. In a Denjoy-Carleman class $\mathcal{Q}_{M}$, closure under differentiation is equivalent to the axiom 2.1(1) of closure under division by a coordinate - the converse of Remark 2.2(1) is a consequence of the fundamental theorem of calculus:

$$
f\left(x_{1}, \ldots, x_{n}\right)-f\left(x_{1}, \ldots, 0, \ldots, x_{n}\right)=x_{i} \int_{0}^{1} \frac{\partial f}{\partial x_{i}}\left(x_{1}, \ldots, t x_{i}, \ldots, x_{n}\right) d t
$$

(where 0 in the left-hand side is in the $i$ th place).

According to the Denjoy-Carleman theorem, the class $\mathcal{Q}_{M}$ is quasianalytic (axiom $2.1(3)$ ) if and only if the assumption (b) holds [12, Thm. 1.3.8]. 
Closure of a Denjoy-Carleman class $\mathcal{Q}_{M}$ under composition is due to Roumieu [22] and closure under inverse to Komatsu [14]; see [4] for simple proofs. A DenjoyCarleman class $\mathcal{Q}_{M}$ satisfying the assumptions (a) and (b) above is thus a quasianalytic class, in the sense of Definition 2.1.

If $\mathcal{Q}_{M}, \mathcal{Q}_{N}$ are Denjoy-Carleman classes, then $\mathcal{Q}_{M}(U) \subseteq \mathcal{Q}_{N}(U)$, for all $U$, if and only if $\sup \left(M_{k} / N_{k}\right)^{1 / k}<\infty$ (see [23, §1.4]); in this case, we write $\mathcal{Q}_{M} \subseteq \mathcal{Q}_{N}$. For any given Denjoy-Carleman class $\mathcal{Q}_{M}$, there is a function in $\mathcal{Q}_{M}((0,1))$ which is nowhere in any given smaller class [13, Thm.1.1]

2.2. Shifted Denjoy-Carleman classes. Given $M=\left(M_{j}\right)_{j \in \mathbb{N}}$ and a positive integer $p$, let $M^{(p)}$ denote the sequence $M_{j}^{(p)}:=M_{p j}$.

If $M$ is logarithmically convex, then $M^{(p)}$ is logarithmically convex:

$$
\frac{M_{k p}}{M_{(k-1) p}}=\frac{M_{k p}}{M_{k p-1}} \cdots \frac{M_{k p-p+1}}{M_{k p-p}} \leq \frac{M_{k p+p}}{M_{k p+p-1}} \cdots \frac{M_{k p+1}}{M_{k p}}=\frac{M_{(k+1) p}}{M_{k p}} .
$$

Therefore, if $\mathcal{Q}_{M}$ is a Denjoy-Carleman class, then so is $\mathcal{Q}_{M(p)}$. Clearly, $\mathcal{Q}_{M} \subseteq$ $\mathcal{Q}_{M^{(p)}}$. Moreover, the assumption (a) above for $\mathcal{Q}_{M}$ immediately implies the same condition for $\mathcal{Q}_{M^{(p)}}$. In general, however, it is not true that assumption (b) (i.e., the quasianalyticity axiom (3)) for $\mathcal{Q}_{M}$ implies (b) for $\mathcal{Q}_{M^{(p)}}$ [20, Example 6.6].

In particular, in general, $\mathcal{Q}_{M(p)} \supsetneq \mathcal{Q}_{M}$. Moreover, $\mathcal{Q}_{M^{(2)}}$ is the smallest DenjoyCarleman class containing all $g \in \mathcal{C}^{\infty}(\mathbb{R})$ such that $g\left(t^{2}\right) \in \mathcal{Q}_{M}(\mathbb{R})$ [20, Rmk. 6.2].

\section{QuASIANALYTIC CONTINUATION}

The goal of this section is to prove Theorem 1.3. We begin with three lemmas that strengthen results in [1, Sect. 4].

Let $\mathcal{F}_{a}$ denote the ring of formal power series centred at a point $a \in \mathbb{R}^{m}$; thus $\mathcal{F}_{a} \cong \mathbb{R} \llbracket x_{1}, \ldots, x_{m} \rrbracket$. If $U$ is open in $\mathbb{R}^{m}$ and $f \in \mathcal{C}^{\infty}(U)$, then $\hat{f}_{a} \in$ $\mathcal{F}_{a}$ denotes the formal Taylor expansion of $f$ at a point $a \in U$; i.e., $\hat{f}_{a}(x)=$ $\sum_{\alpha \in \mathbb{N}^{m}}\left(\partial^{|\alpha|} f / \partial x^{\alpha}\right)(a) x^{\alpha} / \alpha$ ! (likewise for a $\mathcal{C}^{\infty}$ mapping $\left.U \rightarrow \mathbb{R}^{n}\right)$.

Let $\mathcal{Q}$ denote a quasianalytic class (Definition 2.1)

Lemma 3.1. Let $V, W$ denote open neighbourhoods of the origin in $\mathbb{R}^{m}$, with coordinate systems $x=\left(x_{1}, \ldots, x_{m}\right), y=\left(y_{1}, \ldots, y_{m}\right)$, respectively. (Assume $V$ is chosen so that every coordinate hyperplane $\left(x_{i}=0\right)$ is connected). Let $\psi: V \rightarrow W$ denote a $\mathcal{Q}$-mapping such that the Jacobian determinant $\operatorname{det}(\partial \psi / \partial x)$ is a monomial times an invertible factor in $\mathcal{Q}(V)$. Let $f \in \mathcal{Q}(V)$ and let $H \in \mathcal{F}_{0}$ be a formal power series centred at $0 \in W$, such that $\hat{f}_{0}=H \circ \hat{\psi}_{0}$. Then, for all $\beta \in \mathbb{N}^{m}$, there exists $f_{\beta} \in \mathcal{Q}(V)$ such that $f_{0}=f$ and

(1) for all $a \in \psi^{-1}(0)$ and all $\beta \in \mathbb{N}^{m}$,

$$
\hat{f}_{\beta, a}=\frac{\partial^{|\beta|} H}{\partial y^{\beta}} \circ \hat{\psi}_{a}
$$

(2) for all $a \in V, \hat{f}_{a}=H_{a} \circ \hat{\psi}_{a}$, where $H_{a} \in \mathcal{F}_{\psi(a)}$ denotes the formal power series

$$
H_{a}:=\sum_{\beta \in \mathbb{N}^{m}} \frac{f_{\beta}(a)}{\beta !} y^{\beta}
$$


(3) each $f_{\beta}, \beta \in \mathbb{N}^{m}$, and therefore also $H_{a} \in \mathcal{F}_{\psi(a)}$ (as a function of a) is constant on connected components of the fibres of $\psi$;

(4) if $H$ is independent of some variable $y_{j}$, then $H_{a}$ is independent of $y_{j}$, for all $a \in V$.

Proof. The lemma with items (2),(3) is a restatement of [1, Thm. 4.1] (the proof of (3) in the latter, in fact, uses a simpler version [1, Lemma 4.2] of Lemma 3.2 below) and item (1) is contained in the proof of [1, Thm. 4.1].

To prove (4): $H$ is independent of some variable $y_{j}$ if and only if $\partial^{|\beta|} H / \partial y^{\beta}=0$ whenever $\beta=\left(\beta_{1}, \ldots, \beta_{m}\right)$ with $\beta_{j} \neq 0$. By (3.1), the latter condition implies that that $\hat{f}_{\beta, 0}=0$ whenever $\beta_{j} \neq 0$; i.e, that $f_{\beta}=0$ whenever $\beta_{j} \neq 0$ (by quasianalyticity). Therefore, if $H$ is independent of $y_{j}$, then, for every $a, f_{\beta}(a)=0$ whenever $\beta_{j} \neq 0$; i.e., $H_{a}$ is independent of $y_{j}$, by (3.2).

Lemma 3.2. Let $\varphi: V \rightarrow W$ denote a $\mathcal{Q}$-mapping, where $W$ is a neighbourhood of 0 in $\mathbb{R}^{n}$ and $V$ is a $\mathcal{Q}$-manifold. Let $f_{\beta} \in \mathcal{Q}(V)$, for all $\beta \in \mathbb{N}^{n}$. Assume that

(1) all $f_{\beta}$ are constant on the fibre $\varphi^{-1}(0)$; i.e.,

$$
H_{a}:=\sum_{\beta \in \mathbb{N}^{n}} \frac{f_{\beta}(a)}{\beta !} y^{\beta}, \quad a \in \varphi^{-1}(0),
$$

is a formal power series $H_{a}=H$ independent of $a$;

(2) for all $a \in \varphi^{-1}(0)$ and $\beta \in \mathbb{N}^{m}$,

$$
\hat{f}_{\beta, a}=\frac{\partial^{|\beta|} H}{\partial y^{\beta}} \circ \hat{\varphi}_{a}
$$

If $K$ is a compact subset of $\varphi^{-1}(0)$, then all $f_{\beta}$ are constant on all fibres of $\varphi$ in some neighbourhood of $K$.

Proof (cf. proofs of [1, Lemma 4.2, Cor. 4.5]). For every $k \in \mathbb{N}$, let

$$
P_{k}:=\left\{(\xi, \eta, \zeta) \in V \times V \times W: \varphi(\xi)=\zeta=\varphi(\eta), f_{\beta}(\xi)=f_{\beta}(\eta),|\beta| \leq k\right\} .
$$

Then the decreasing sequence of closed quasianalytic sets $P_{0} \supset P_{1} \supset P_{2} \supset \ldots$ stabilizes in some neighbourhood of $K$, by topological noetherianity [4, Thm. 6.1]; say, $P_{k}=P_{k_{0}}, k \geq k_{0}$, in such a neighbourhood. It follows that, if $f_{\beta}$ is constant on the fibres of $\varphi$ in a given neighbourhood of $K$, for all $\beta \leq k_{0}$, then $f_{\beta}$ is constant on the fibres of $\varphi$ in some neighbourhood of $K$, for all $\beta$.

Therefore, it is enough to prove the following assertion: given $\beta \in \mathbb{N}^{n}, f_{\beta}$ is constant on the fibres of $\varphi$ over $W$, in some neighbourhood of $K$. The following argument is due to Nowak [19]. Define

$$
P:=\left\{(\xi, \eta, \zeta) \in V \times V \times W: \varphi(\xi)=\zeta=\varphi(\eta), f_{\beta}(\xi) \neq f_{\beta}(\eta)\right\} .
$$

Suppose the assertion is false. Then there is a point $\left(a_{1}, a_{2}, 0\right) \in \bar{P}$ and, by the quasianalytic curve selection lemma (see [4. Thm.6.2]), a quasianalytic $\operatorname{arc}(\xi(t), \eta(t), \zeta(t)) \in V \times V \times W$ such that $(\xi(0), \eta(0), \zeta(0))=\left(a_{1}, a_{2}, 0\right)$ and $(\xi(t), \eta(t), \zeta(t)) \in P$ if $t \neq 0$. Then

$$
\left(f_{\beta} \circ \xi\right)_{0}^{\wedge}=\hat{f}_{\beta, a_{1}} \circ \hat{\xi}_{0}=\frac{\partial^{|\beta|} H}{\partial y^{\beta}} \circ \hat{\varphi}_{a_{1}} \circ \hat{\xi}_{0}=\frac{\partial^{|\beta|} H}{\partial y^{\beta}} \circ(\varphi \circ \xi)_{0}^{\wedge}=\frac{\partial^{|\beta|} H}{\partial y^{\beta}} \circ \hat{\zeta}_{0} .
$$

Likewise, $\left(f_{\beta} \circ \eta\right)_{0}^{\wedge}=\left(\partial^{|\beta|} H / \partial y^{\beta}\right) \circ \hat{\zeta}_{0}$, so that $\left(f_{\beta} \circ \xi\right)_{0}^{\wedge}=\left(f_{\beta} \circ \eta\right)_{0}^{\wedge}$. Since $f_{\beta} \circ \xi, f_{\beta} \circ \eta$ are quasianalytic functions of $t, f_{\beta} \circ \xi=f_{\beta} \circ \eta$; a contradiction. 
Lemma 3.3. Let $\varphi: V \rightarrow W$ denote a $\mathcal{Q}$-mapping, where $W$ is a neighbourhood of 0 in $\mathbb{R}^{n}$ and $V$ is a $\mathcal{Q}$-manifold. Let $K$ be a compact subset of $V$ and let $\{U\}$ be an open covering of $\varphi^{-1}(0) \bigcap K$. Let $H \in \mathcal{F}_{0}$ be a power series centred at $0 \in W$. Suppose there exists $f_{U} \in \mathcal{Q}(U)$, for each $U$, such that $\hat{f}_{U, a}=\hat{\varphi}_{a}^{*}(H)$, for all $a \in \varphi^{-1}(0) \cap U$. Then there exists $f \in \mathcal{Q}\left(V^{\prime}\right)$, where $V^{\prime}$ is a neighbourhood of $\varphi^{-1}(0) \bigcap K$, such that $\hat{f}_{a}=\hat{\varphi}_{a}^{*}(H)$, for all $a \in \varphi^{-1}(0) \bigcap K$.

Proof. Lemma 3.3 generalizes [1, Lemma 4.4] and the proof is the same.

Proof of Theorem [1.3. (1) is a restatement of [1, Prop. 4.6], so we only prove (2) here.

Write $\varphi=\left(\varphi_{1}, \ldots, \varphi_{n}\right)$. Every point of $\varphi^{-1}(0)$ has a neighbourhood $U$ in $V$ with a coordinate system $\left(x_{1}, \ldots, x_{m}\right)$ in which the Jacobian submatrix

is generically of rank $n$.

$$
\frac{\partial\left(\varphi_{1}, \ldots, \varphi_{n}\right)}{\partial\left(x_{1}, \ldots, x_{n}\right)}
$$

Fix such $U$ and define $\psi_{U}: U \rightarrow \mathbb{R}^{m}$ by

$$
\psi_{U}\left(x_{1}, \ldots, x_{m}\right)=\left(\varphi(x), x_{n+1}, \ldots, x_{m}\right) .
$$

Then, for all $a \in \varphi^{-1}(0)$,

$$
\hat{f}_{a}=H \circ \hat{\varphi}_{a}=H_{U} \circ \hat{\psi}_{U, a},
$$

where $H_{U}\left(y_{1}, \ldots, y_{m}\right)=H\left(y_{1}, \ldots, y_{n}\right)$; in particular, the formal power series $H_{U}(y)$ is independent of $y_{n+1}, \ldots, y_{m}$.

We claim there is a proper surjective mapping $\sigma_{U}: Z_{U} \rightarrow U$, where $Z_{U}$ is a $\mathcal{Q}$-manifold of dimension $m$ ( $\sigma_{U}$ is a composite of finitely many blowings-up if $U$ is small enough) such that every point of $Z_{U}$ has a neighbourhood with coordinates $\left(z_{1}, \ldots, z_{m}\right)$ in which the Jacobian determinant $\operatorname{det}\left(\partial\left(\psi_{U} \circ \sigma_{U}\right) / \partial z\right)$ is a monomial in $z$ times an invertible factor. In fact, by resolution of singularities [4, Thm. 5.9], there exists $\sigma_{U}: Z_{U} \rightarrow U$ such that every point of $Z_{U}$ has a neighbourhood with coordinates $\left(z_{1}, \ldots, z_{m}\right)$ in which $\left(\operatorname{det}\left(\partial \psi_{U} / \partial x\right)\right) \circ \sigma_{U}$ and $\operatorname{det}\left(\partial \sigma_{U} / \partial z\right)$ are both monomials times invertible factors, so the claim follows from the chain rule.

Now, $\varphi^{-1}(0)$ is covered by finitely many open sets $U$ as above. Choose compact $K_{U} \subset U$ so that $\varphi^{-1}(0)=\bigcup K_{U}$. Consider the mapping $\sigma: Z \rightarrow V$ from the disjoint union $Z:=\coprod Z_{U}$, where $\sigma$ is given by $\sigma_{U}$ on each $Z_{U}$. Set $\Phi:=\varphi \circ \sigma$. By Lemmas 3.1, 3.2 and 3.3, after shrinking $W$ and the $Z_{U}$ to suitable neighbourhoods of 0 and the $\sigma_{U}^{-1}\left(K_{U}\right)$ (respectively), $\sigma(Z)=\varphi^{-1}(W)$ and there exist $f_{\beta} \in \mathcal{Q}(Z)$, $\beta \in \mathbb{N}^{n}$, such that each $f_{\beta}$ is constant on the fibres of $\Phi$ and, for all $U$ and $a^{\prime} \in Z_{U}$,

$$
\left(f \circ \sigma_{U}\right)_{a^{\prime}}^{\wedge}=H_{a^{\prime}} \circ \hat{\varphi}_{\sigma_{U}\left(a^{\prime}\right)} \circ \hat{\sigma}_{U, a^{\prime}},
$$

where

$$
H_{a^{\prime}}=\sum_{\beta \in \mathbb{N}^{n}} \frac{f_{\beta}\left(a^{\prime}\right)}{\beta !} y^{\beta}
$$

$\left(\hat{\sigma}_{U, a^{\prime}}\right.$ can be defined using local coordinates $\left(z_{1}, \ldots, z_{m}\right)$ in a neighbourhood of any point of $\left.Z_{U}\right)$. So we can set $H_{b}:=H_{a^{\prime}}$, where $a^{\prime} \in \Phi^{-1}(b)$, for any $b \in W$.

Finally, we note that the power series homomorphism $\mathcal{F}_{\varphi\left(a^{\prime}\right)} \rightarrow \mathcal{F}_{a^{\prime}}$ given by composition with $\hat{\sigma}_{U, a^{\prime}}$ is injective, for any $a^{\prime} \in Z_{U}$ as above. This is a consequence of the fact that, with respect to local coordinates $\left(z_{1}, \ldots, z_{m}\right)$ at $a^{\prime}$, the determinant of the Jacobian matrix of formal derivatives $\partial \hat{\sigma}_{U, a^{\prime}} / \partial z$ is a nonzero formal power 
series, by the quasianalyticity axiom and the fact that $\sigma_{U}$ is generically of rank $m$. We can therefore conclude from (3.4) that $\hat{f}_{a}=H_{b} \circ \hat{\varphi}_{a}$, for all $b \in W$ and $a \in \varphi^{-1}(b)$, as required.

\section{Proofs of the main theorems}

In this section, we prove Theorems 1.1, 1.2 and 1.6, using the regularity estimates of Theorem 1.4 and Corollary 1.5, which are proved in Section 5. For the composite function theorems we also need the quasianalytic continuation theorem 1.3 .

4.1. Composite function theorems. We can assume that $W$ is an open neighbourhood of $0 \in \mathbb{R}^{n}$, in both Theorems 1.1 and 1.2

Remark 4.1. Theorems 1.1 and 1.2 are equivalent (for given $\operatorname{dim} V \geq n$ ): With the hypotheses of Theorem 1.1 it follows from the quasianalytic continuation theorem 1.3 that there is a neighbourhood of $a$ on which $f$ is formally composite with $\sigma$. It is then easy to see that Theorem 1.2 implies 1.1. Conversely, assuming Theorem 1.1. it follows from the hypotheses of Theorem 1.2 that each $a \in \varphi^{-1}(0)$ has a relatively compact neighbourhood $U$ on which $f=g \circ \varphi$, where $g \in \mathcal{Q}_{M^{(p)}}(\varphi(\bar{U}))$, for some $p$, and the conclusion of Theorem 1.2 is a simple consequence.

Proof of Theorem 1.2. It follows from Theorem 1.3 that, after shrinking $W$ to a suitable neighbourhood of $b, f$ is formally composite with $\varphi$. By a quasianalytic version [18] of Glaeser's theorem [10], $f=g \circ \varphi$, where $g \in \mathcal{C}^{\infty}(W)$. The theorem then follows from Theorem 1.4 or Corollary 1.5.

Remark 4.2. Theorems 1.2 and 1.3 do not hold under the weaker assumption that $\varphi$ is semiproper and generically submersive, as in Glaeser's theorem, unless we admit the possibility of also shrinking $V$ to a suitable neighbourhood of $\varphi^{-1}(0)$. (In general, the conclusion of Theorem[1.3(2) holds for the restriction of $\varphi$ to some neighbourhood of any compact $K \subset \varphi^{-1}(0)$.) Corollary 1.5 nevertheless holds for $\varphi$ semiproper because $f$ is assumed to be a composite $g \circ \varphi$, where $g$ is $\mathcal{C}^{\infty}$.

\subsection{Model theory.}

Remark 4.3. To prove Theorem 1.6, we use the fact that, for a given quasianalytic class $\mathcal{Q}$, any closed sub-quasianalytic subset $X$ of $\mathbb{R}^{n}$ (the analogue in class $\mathcal{Q}$ of a subanalytic set) is the image of a proper $\mathcal{Q}$-mapping $\sigma: Z \rightarrow \mathbb{R}^{n}$, where $Z$ is a $\mathcal{Q}$-manifold of dimension $=\operatorname{dim} X$. This can be proved in the same way as the proof in 2] for a subanalytic set: First, $X$ is (at least locally) the image by a proper $\mathcal{Q}$-mapping of a semi-quasianalytic subset $Y$ of $\mathbb{R}^{q}$, for some $q$ (essentially by definition). Moreover, we can assume that $\operatorname{dim} Y=\operatorname{dim} X$, by the fibre-cutting lemma [2, Lemma 3.6]. It is easy to see that a closed semi-quasianalytic set is a proper image of a closed quasianalytic set (the $\mathcal{Q}$-analogue of an analytic set) of the same dimension [2, Prop. 3.12], and the result then follows from resolution of singularities.

Proof of Theorem 1.6. Let $B^{n} \subset \mathbb{R}^{n}$ denote the closed unit ball, and define $\rho$ : $\mathbb{R}^{n} \rightarrow B^{n}$ by

$$
\rho(x):=\frac{x}{\sqrt{1+\|x\|^{2}}}, \quad x \in \mathbb{R}^{n},
$$

where $\|x\|^{2}:=x_{1}^{2}+\cdots x_{n}^{2}$. Then $\rho$ is an analytic isomorphism onto the open ball. Let $g:=f \circ \rho^{-1}: \rho(W) \rightarrow \mathbb{R} \subset S^{1}$ (where $S^{1}$ is the compactification of $\mathbb{R}$ by 
adding a single point at infinity), and let $X$ denote the closure of the graph of $g$ in $B^{n} \times S^{1} \subset \mathbb{R}^{n} \times S^{1}$. Since $f$ is definable, $X$ is a compact sub-quasianalytic subset of $\mathbb{R}^{n} \times S^{1}$ (of class $\mathcal{Q}_{M}$ ).

By Remark 4.3, there is a compact $\mathcal{Q}_{M}$-manifold $Z$, where $\operatorname{dim} Z=\operatorname{dim} X=n$, and a $\mathcal{Q}_{M}$-mapping $\sigma: Z \rightarrow \mathbb{R}^{n} \times S^{1}$ such that $\sigma(Z)=X$. Write $\sigma=\left(\sigma_{1}, \sigma_{2}\right)$ with respect to the projections to $\mathbb{R}^{n}$ and $S^{1}$. Then $g \circ \sigma_{1}=\sigma_{2}$ on $\sigma^{-1}\left(\rho(W) \times S^{1}\right) \subset Z$, so that $g \circ \sigma_{1}$ is of class $\mathcal{Q}_{M}$ on $\sigma^{-1}\left(\rho(W) \times S^{1}\right)$. By Corollary 1.5, $g \in \mathcal{Q}_{M^{(p)}}(\rho(W))$, for some $p$. Therefore, $f \in \mathcal{Q}_{M^{(p)}}(W)$, since $f=g \circ \rho$ and $\rho$ is an analytic isomorphism from $\mathbb{R}^{n}$ to the open ball.

\section{Regularity estimates}

Proof of Theorem 1.4. We can assume that $K=[-r, r]^{n} \subset \mathbb{R}^{n}$ for some $r>0$. Let $\operatorname{Jac} \sigma$ denote the Jacobian matrix $(\partial \sigma / \partial x)$, and write $\operatorname{det} \operatorname{Jac} \sigma=x^{\gamma} \Delta(x)$, where $\Delta$ is nowhere vanishing. Let $T=\left(T_{j i}\right):=(\operatorname{Jac} \sigma)^{*} / \Delta(x)$, where $(\operatorname{Jac} \sigma)^{*}$ denotes the adjugate matrix of Jac $\sigma$. Let $\left(\partial_{x_{i}}\right)$ denote the (column) vector with entries $\partial_{x_{i}}:=\partial / \partial x_{i}, i=1, \ldots, n$. Thus,

$$
\left(\partial_{x_{i}}\right)=\operatorname{Jac} \sigma \cdot\left(\partial_{y_{j}}\right) \quad \text { or } \quad\left(\partial_{y_{j}}\right)=\frac{1}{x^{\gamma}} \cdot T \cdot\left(\partial_{x_{i}}\right) .
$$

Since $f$ and $\sigma$ are of class $\mathcal{Q}_{M}$, there is a constant $B \geq 1$ such that

$$
\begin{aligned}
& \left|f^{(\alpha)}\right| \leq B^{|\alpha|} \alpha ! M_{|\alpha|} \\
& \left|T_{j i}^{(\alpha)}\right| \leq B^{|\alpha|} \alpha ! M_{|\alpha|+|\gamma|}, \quad i, j=1, \ldots, n,
\end{aligned}
$$

on $K$, for all $\alpha \in \mathbb{N}^{n}$.

Set $p:=2|\gamma|+1$. We will show that

$$
\left|\frac{\partial^{|\beta|} g}{\partial y^{\beta}}\right| \leq\left(n^{2} p \xi E^{p}\right)^{|\beta|} \beta ! M_{p|\beta|}
$$

on $\sigma(K)$, for all $\beta \in \mathbb{N}^{n}$, where $\xi$ is a fixed positive constant and $E>\max \{1, B\}$ is big enough that

$$
\sum_{\lambda \in \mathbb{N}^{n}}\left(\frac{B}{E}\right)^{|\lambda|}<\xi
$$

The estimate (1.1) follows. In the argument below, all estimates are understood to mean "on $K$ " or "on $\sigma(K)$ ", as the case may be, and we will not say this explicitly.

Claim 5.1. For each $\beta \in \mathbb{N}^{n}$,

$$
\left|\frac{\partial^{|\alpha|}\left(g^{(\beta)} \circ \sigma\right)}{\partial x^{\alpha}}\right| \leq(n \xi)^{|\beta|} E^{p|\beta|+|\alpha|} \Gamma(\alpha, \beta) M_{p_{\beta}(\alpha)},
$$

for all $\alpha \in \mathbb{N}^{n}$, where $p_{\beta}(\alpha):=p|\beta|+|\alpha|$ and

$$
\Gamma(\alpha, \beta)=\alpha ! \cdot \prod_{j=1}^{|\beta|} \max _{1 \leq i \leq n}\left\{\alpha_{i}+j\left(\gamma_{i}+1\right)\right\} .
$$

Note that $p_{\beta}(0)=p|\beta|$, and that

$$
\Gamma(0, \beta)=\prod_{j=1}^{|\beta|} \max _{1 \leq i \leq n}\left\{j\left(\gamma_{i}+1\right)\right\} \leq \prod_{j=1}^{|\beta|} p j=p^{|\beta|}|\beta| ! \leq(n p)^{|\beta|} \beta !
$$


Therefore, (5.4) in the case that $\alpha=0$ implies (5.3); i.e., the estimate (1.1) follows from Claim 5.1.

We will prove the claim by induction on $|\beta|$. Note that $\Gamma(\alpha, 0)=\alpha$ !. The claim is therefore true when $\beta=0$, because in this case (5.4) reduces to (5.2) (recall that $E>B$ ). Fix a multiindex $\tilde{\beta}$, where $|\tilde{\beta}|>0$. By induction, we assume the claim holds for all $\beta$ such that $|\beta|<|\tilde{\beta}|$.

Without loss of generality, there exists $\beta \in \mathbb{N}^{n}$ such that $\tilde{\beta}=\beta+(1)$. By (5.1) and the fundamental theorem of calculus (used $|\gamma|$ times),

$$
\frac{\partial g^{(\beta)}}{\partial y_{1}} \circ \sigma=\int_{[0,1]|\gamma|} \sum_{i=1}^{n} \frac{\partial^{|\gamma|}}{\partial x^{\gamma}}\left(T_{1 i} \cdot \frac{\partial g^{(\beta)} \circ \sigma}{\partial x_{i}}\right)\left(\prod_{j=1}^{\gamma_{k}} t_{k, j} x_{k}\right) Q_{0}(t) d t
$$

where $t:=\left(t_{1}, \ldots, t_{n}\right), t_{k}:=\left(t_{k, 1}, \ldots, t_{k, \gamma_{k}}\right)$, and $Q_{0}(t):=\prod_{k=1}^{n} \prod_{j=1}^{\gamma_{k}} t_{k, j}^{\gamma_{k}-j}$. It follows that, for all $\alpha \in \mathbb{N}^{n}$,

$$
\begin{aligned}
\left|\frac{\partial^{|\alpha|}\left(g^{(\tilde{\beta})} \circ \sigma\right)}{\partial x^{\alpha}}\right| & =\left|\frac{\partial^{|\alpha|}}{\partial x^{\alpha}}\left(\frac{\partial g^{(\beta)}}{\partial y_{1}} \circ \sigma\right)\right| \\
& \leq \int_{[0,1]|\gamma|} \sum_{i=1}^{n}\left|\frac{\partial^{|\gamma|+|\alpha|}}{\partial x^{\gamma+\alpha}}\left(T_{1 i} \cdot \frac{\partial g^{(\beta)} \circ \sigma}{\partial x_{i}}\right)\right| Q_{\alpha}(t) d t \\
& \leq \int_{[0,1]|\gamma|} \sum_{i=1}^{n} \sum_{\lambda+\delta=\alpha+\gamma}\left(\begin{array}{c}
\alpha+\gamma \\
\lambda
\end{array}\right)\left|\frac{\partial^{|\lambda|} T_{1 i}}{\partial x^{\lambda}} \cdot \frac{\partial^{|\delta|+1} g^{(\beta)} \circ \sigma}{\partial x^{\delta} \partial x_{i}}\right| Q_{\alpha}(t) d t
\end{aligned}
$$

where $Q_{\alpha}(t)=\prod_{k=1}^{n} \prod_{j=1}^{\gamma_{k}} t_{k, j}^{\gamma_{k}+\alpha_{k}-j}$, so that:

$$
\int_{[0,1]|\gamma|} Q_{\alpha}(t) d t=\frac{\alpha !}{(\alpha+\gamma) !}
$$

Moreover, by (5.2) and (5.4),

$$
\begin{aligned}
\left|\frac{\partial^{|\lambda|} T_{k, i}}{\partial x^{\lambda}}\right| & \leq B^{|\lambda|} \lambda ! M_{|\lambda|+|\gamma|} \\
\left|\frac{\partial^{|\delta|+1} g^{(\beta)} \circ \sigma}{\partial x^{\delta} \partial x_{i}}\right| & \leq(n \xi)^{|\beta|} E^{p|\beta|+|\delta|+1} \Gamma(\delta+(i), \beta) M_{p_{\beta}(\delta+(i))}
\end{aligned}
$$

Since $p_{\beta}(\delta+(i))+|\lambda|+|\gamma|=p|\beta|+2|\gamma|+1+|\alpha|=p_{\tilde{\beta}}(\alpha)$, the preceding inequalities imply (using log-convexity) that

$$
\begin{aligned}
\left|\frac{\partial^{|\lambda|} T_{k, i}}{\partial x^{\lambda}} \cdot \frac{\partial^{|\delta|+1} g^{(\beta)} \circ \sigma}{\partial x^{\delta} \partial x_{i}}\right| & \leq(n \xi)^{|\beta|} B^{|\lambda|} E^{p|\beta|+|\delta|+1} \lambda ! \Gamma(\delta+(i), \beta) M_{p_{\tilde{\beta}}(\alpha)} \\
& \leq(n \xi)^{|\beta|} E^{p|\tilde{\beta}|+|\alpha|}\left(\frac{B}{E}\right)^{|\lambda|} \lambda ! \Gamma(\delta+(i), \beta) M_{p_{\tilde{\beta}}(\alpha)}
\end{aligned}
$$

We conclude from (5.6) and (5.7) that

$$
\begin{aligned}
\left|\frac{\partial^{|\alpha|}\left(g^{(\tilde{\beta})} \circ \sigma\right)}{\partial x^{\alpha}}\right| & \leq \sum_{i=1}^{n} \sum_{\lambda+\delta=\alpha+\gamma}\left(\begin{array}{c}
\alpha+\gamma \\
\lambda
\end{array}\right) \frac{\alpha ! \lambda ! \Gamma(\delta+(i), \beta)}{(\alpha+\gamma) !}(n \xi)^{|\beta|} E^{p|\tilde{\beta}|+|\alpha|}\left(\frac{B}{E}\right)^{|\lambda|} M_{p_{\tilde{\beta}}(\alpha)} \\
& \leq \xi^{|\beta|}\left(n E^{p}\right)^{|\tilde{\beta}|} E^{|\alpha|} M_{p_{\tilde{\beta}}(\alpha)} \sum_{\lambda+\delta=\alpha+\gamma} \frac{\alpha !}{\delta !} \max _{1 \leq i \leq n}\{\Gamma(\delta+(i), \beta)\}\left(\frac{B}{E}\right)^{|\lambda|} .
\end{aligned}
$$


Now, from the definition of $\Gamma(\alpha, \beta)$ and the fact that $\delta \leq \alpha+\gamma$, we obtain

$$
\begin{aligned}
\frac{\alpha !}{\delta !} \max _{1 \leq i \leq n}\{\Gamma(\delta+(i), \beta)\} & =\alpha ! \max _{1 \leq i \leq n}\left\{\frac{(\delta+(i)) !}{\delta !} \prod_{j=1}^{|\beta|} \max _{1 \leq k \leq n}\left\{\delta_{k}+(1)+j\left(\gamma_{k}+1\right)\right\}\right\} \\
& \leq \alpha ! \max _{1 \leq i \leq n}\left\{\frac{(\delta+(i)) !}{\delta !} \prod_{j=1}^{|\beta|} \max _{1 \leq k \leq n}\left\{\alpha_{k}+\gamma_{k}+1+j\left(\gamma_{k}+1\right)\right\}\right\} \\
& \leq \alpha ! \max _{1 \leq i \leq n}\left\{\frac{(\delta+(i)) !}{\delta !}\right\} \prod_{j=2}^{|\tilde{\beta}|} \max _{1 \leq i \leq n}\left\{\alpha_{i}+j\left(\gamma_{i}+1\right)\right\} \\
& \leq \alpha ! \max _{1 \leq i \leq n}\left\{\alpha_{i}+\gamma_{i}+1\right\} \prod_{j=2}^{|\tilde{\beta}|} \max _{1 \leq i \leq n}\left\{\alpha_{i}+j\left(\gamma_{i}+1\right)\right\} \\
& =\alpha ! \prod_{j=1}^{|\tilde{\beta}|} \max _{1 \leq i \leq n}\left\{\alpha_{i}+j\left(\gamma_{i}+1\right)\right\}=\Gamma(\alpha, \tilde{\beta}) .
\end{aligned}
$$

We conclude, therefore, that

$$
\begin{aligned}
\left|\frac{\partial^{|\alpha|}\left(g^{(\tilde{\beta})} \circ \sigma\right)}{\partial x^{\alpha}}\right| & \leq \xi^{|\beta|}\left(n E^{p}\right)^{|\tilde{\beta}|} E^{|\alpha|} \Gamma(\alpha, \tilde{\beta}) M_{p_{\tilde{\beta}}(\alpha)} \sum_{\lambda \in \mathbb{N}^{n}}\left(\frac{B}{E}\right)^{|\lambda|} \\
& \leq\left(n \xi E^{p}\right)^{|\tilde{\beta}|} E^{|\alpha|} \Gamma(\alpha, \tilde{\beta}) M_{p_{\tilde{\beta}}(\alpha)},
\end{aligned}
$$

as required for the estimate (1.1).

It remains to show that we can get the estimate (1.2) if we make the stronger assumption that $\mathcal{Q}_{M}$ is closed under differentiation (i.e., $\mathcal{Q}_{M}$ satisfies the assumption (a) of 2.1). Under this assumption, we can strengthen the second inequality of (5.2) to

$$
\left|T_{j i}^{(\alpha)}\right| \leq B^{|\alpha|} \alpha ! M_{|\alpha|}, \quad i, j=1, \ldots, n,
$$

and the required estimate (1.2) can be obtained exactly as above.

Remark 5.2. It is possible to prove Theorem 1.4 also by an argument similar to that of Chaumat and Chollet [8, Sect. III]. Indeed, in the notation of [8] (for a reader familiar with the latter), the estimate in [8, Lemma III.4] can be improved to

$$
\left|\frac{\partial}{\partial x^{S}}\left(T_{K, L}\right)(x)\right| \leq C_{3}^{l-s+1} \frac{(s+l) !}{k !} M_{p s}
$$

where $p=2|\gamma|+1$, and this can be used in a calculation similar to that of [8].

Proof of Corollary 1.5. If $\operatorname{dim} V=\operatorname{dim} W$, then, by resolution of singularities, there is a proper surjective mapping $\sigma: Z \rightarrow V$ of class $\mathcal{Q}_{M}(\operatorname{dim} Z=\operatorname{dim} V)$ such that $\operatorname{det}(\partial(\varphi \circ \sigma) / \partial x)$ is locally a monomial times an invertible factor (as in the proof of Theorem [1.3), so the result follows from Theorem 1.4.

In general, we can reduce to the preceding special case by using a mapping analogous to (3.3), locally. 


\section{REFERENCES}

1. A. Belotto da Silva, I. Biborski and E. Bierstone, Solutions of quasianalytic equations, Selecta Math. New Ser. (to appear), arXiv:1605.01425 2 [math.CV] (25 pages).

2. E. Bierstone and P.D. Milman, Semianalytic and subanalytic sets, Inst. Hautes Etudes Sci. Publ. Math. 67 (1988), 5-42

3. E. Bierstone and P.D. Milman, Canonical desingularization in characteristic zero by blowing up the maximum strata of a local invariant, Invent. Math. 128 (1997), 207-302.

4. E. Bierstone and P.D. Milman, Resolution of singularities in Denjoy-Carleman classes, Selecta Math. (N.S.) 10 (2004), 1-28.

5. E. Bierstone, P.D. Milman and G. Valette, Arc-quasianalytic functions, Proc. Amer. Math. Soc. 143 (2015), 3915-3925.

6. E. Borel, Sur la généralisation du prolongement analytique, C. R. Acad. Sci. Paris 130 (1900), $1115-1118$.

7. T. Carleman, Les Fonctions Quasi-analytiques, Collection Borel, Gauthier-Villars, Paris, 1926.

8. J. Chaumat and A. Chollet, Sur la division et la composition dans des classes ultradifférentiables, Studia Math. 136 (1999), 49-70.

9. A. Denjoy, Sur les fonctions quasi-analytiques de variable reélle, C. R. Acad. Sci. Paris $\mathbf{1 7 3}$ (1921), 3120-1322.

10. G. Glaeser, Fonctions composées différentiables, Ann. of Math. 77 (1963), 193-209.

11. J. Hadamard, Lectures on Cauchy's Problem in LInear Partial Differential Equations, Yale Univ. Press, New Haven, 1923.

12. L. Hörmander, The Analysis of Linear Partial Differential Operators I, Springer-Verlag, Berlin-Heidelberg-New York, 1983.

13. E. Jaffe, Pathological phenomena in Denjoy-Carleman classes, Can. J. Math. 68 (2016), 88108.

14. H. Komatsu, The implicit function theorem for ultradifferentiable mappings, Proc. Japan Acad. 55 (1979), 69-72.

15. B. Malgrange, Frobenius avec singularités, 2. Le cas général, Invent. Math. 39 (1977), 67-89.

16. S. Mandelbrojt, Séries Adhérentes, Régularisation des Suites, Applications, Collection Borel, Gauthiers-Villars, Paris, 1952.

17. C. Miller, Infinite differentiability in polynomially bounded o-minimal structures, Proc. Amer. Math. Soc. 123 (1995), 2551-2555.

18. K.J. Nowak, A note on Bierstone-Milman-Pawtucki's paper "Composite differentiable functions", Ann. Polon. Math. 102 (2011), 293-299.

19. K.J. Nowak, On division of quasianalytic function germs, Int. J. Math. 13 (2013), 1-5.

20. K.J. Nowak, Quantifier elimination in quasianalytic structures via non-standard analysis, Ann. Polon. Math. 114 (2015), 235-267.

21. J.-P. Rolin, P. Speissegger and A. J. Wilkie, Quasianalytic Denjoy-Carleman classes and o-minimality, J. Amer. Math. Soc. 16 (2003), 751-777.

22. C. Roumieu, Ultradistributions définies sur $\mathbb{R}^{n}$ et sur certaines classes de variét'es différentiables, J. Analyse Math. 10 (1962-63), 153-192.

23. V. Thilliez, On quasianalytic local rings, Expo. Math. 26 (2008), 1-23.

(A. Belotto) Université Paul Sabatier, Institut de Mathématiques de Toulouse, 118 route de Narbonne, F-31062 Toulouse Cedex 9, France

E-mail address: andre.belotto_da_silva@math.univ-toulouse.fr

(E. Bierstone and M. Chow) University of Toronto, Department of Mathematics, 40

St. George Street, Toronto, ON, Canada M5S 2E4

E-mail address: bierston@math.toronto.edu

E-mail address: mikey.chow@mail.utoronto.ca 